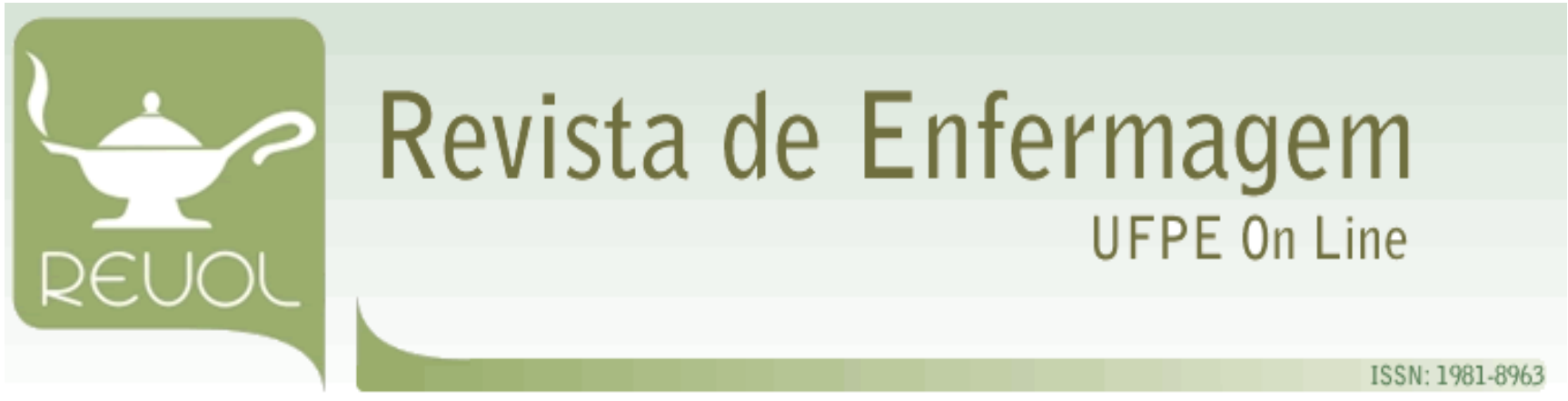

ORIGINAL ARTICLE

\title{
QUALITY OF LIFE OF PATIENTS AT THE REFERRAL EMERGENCY UNIT OF THE
} CLINICS HOSPITAL/UNICAMP

\author{
QUALIDADE DE VIDA DE PACIENTES DA UNIDADE DE EMERGÊNCIA REFERENCIADA DO \\ HOSPITAL DE CLÍNICAS/UNICAMP
}

\section{CALIDAD DE VIDA DE LOS PACIENTES DE LA UNIDAD DE EMERGENCIA DE REFERENCIA EN EL HOSPITAL DE CLÍNICAS/UNICAMP}

Ana Carolina Gaban ${ }^{1}$, Kátia Stancato ${ }^{2}$

\begin{abstract}
Objective: to understand the quality of life $(\mathrm{QOL})$ of patients treated at the referenced Emergency Unit of the Clinics Hospital of the State University of Campinas. Method: quantitative, cross-sectional and descriptive study, using the abbreviated version of the World Health Organization Quality of Life assessment, the WHOQOL-bref in 100 patients admitted to the service. The characteristics of QOL studies cover four areas - physical, psychological, environmental and social relationships. Results: the population's average age was 40.0 years, ranging from age 18.0 to 83.0 years. There was a female predominance, $71.0 \%$ women and $29.0 \%$ men. We observed the highest score in the psychological domain (66.0) (SD: 17.0), and the environmental domain showed the lowest (55.0) (SD: 15.0), which includes availability and quality of health services. The social relationships domain had a higher maximum score, scoring 100, followed by the physical domain (96), psychological (92) and environmental (91). Conclusion: the identification of problems and needs presented by patients on site, by the QOL assessment at the moment of their admission to the service in their physical, social, psychological aspects and their relationship with the environment, allows nurses to focus their assistance and direct patients to other services (psychiatry, nutritionist, social worker, physiotherapy, others). This way, without mischaracterizing the treatment provided by the service, treatment can be offered according to the average of the parameters of QOL presented by the patients, these being the ultimate consumers of the health service. Key words: quality of life; patients; epidemiology.
\end{abstract}

\section{RESUMO}

Objetivo: conhecer a qualidade de vida (QV) dos pacientes atendidos na Unidade de Emergência Referenciada do Hospital de Clínicas da Universidade Estadual de Campinas. Método: estudo quantitativo, transversal e descritivo, utilizando a escala abreviada de qualidade de vida da Organização Mundial de Saúde, o WHOQOL-bref, em 100 pacientes. 0 projeto foi aprovado pelo Comitê de Ética em Pesquisa da Faculdade de Ciências Médicas da Universidade Estadual de Campinas, parecer $n^{\circ} 1036 / 2009$. Resultados: a população apresentou idade média de 40,0 anos, com variação da faixa etária de 18,0 a 83,0 anos. Houve predomínio do sexo feminino, $71,0 \%$ mulheres e $29,0 \%$ homens. Observou-se o maior escore no domínio psicológico $(66,0)$ (DP:17,0), e o domínio ambiental apresentou o menor $(55,0)$ (DP: 15,0), que inclui disponibilidade e qualidade dos serviços de saúde. O domínio relações sociais apresentou maior pontuação máxima, com escore 100, seguidos pelo domínio físico (96), psicológico (92) e ambiental (91). Conclusão: a identificação dos problemas e necessidades apresentados pelos pacientes no local, pela OV apresentada no momento da admissão ao serviço, em seus aspectos físicos, sociais, psicológicos e relações com o meio ambiente, possibilita ao enfermeiro o enfoque assistencial e direcionamento a outros serviços (psiquiatria, nutricionista, assistência social, fisioterapia, outros). Assim, sem descaracterizar o atendimento do serviço, há o acréscimo do olhar terapêutico de acordo com a média dos parâmetros de qualidade de vida apresentados pelos pacientes, sendo estes, os consumidores finais do serviço de saúde. Descritores: qualidade de vida; pacientes; epidemiologia.

\section{RESUMEN}

Objetivo: conocer la calidad de vida (QOL) de los pacientes atendidos en la Unidad de Emergencia de Referencia en Hospital de Clínicas en la Universidad Estatal de Campinas. Método: cuantitativo, transversal, descriptivo, utilizando la escala abreviada de la calidad de vida de la Organización Mundial de la Salud, el WHOQOL-bref en 100 pacientes ingresados en el sitio. Las características de los estudios de la calidad de vida cubren cuatro áreas - física, la relación psicológica, social y medio ambiente. Resultados: la edad media de la población fue de 40,0 años, que van desde los 18,0 a 83,0 años. Hubo predominio del sexo femenino, las mujeres $71,0 \%$ y $29,0 \%$ hombres. Hemos observado el mayor puntaje en el dominio psicológico $(66,0)(\mathrm{DE}: 17,0)$, y del medio ambiente mostraron la más baja $(55,0)(\mathrm{DE}: 15,0)$, que incluye la disponibilidad y la calidad de los servicios de salud. El dominio de las relaciones sociales tenían una puntuación máxima más alta, anotando 100, seguido por el dominio físico (96), psicológicos (92) y ambientales (91). Conclusión: la identificación de problemas y necesidades que presentan los pacientes en el sitio, presentado por la QOL al ingresar en el servicio en términos de relación física, social, psicológica y con el medio ambiente, permite a las enfermeras para enfocar la asistencia y orientación a otros servicios (psiquiatría, especialista en dietética, trabajo social, fisioterapia, otro). Por lo tanto, sin estropear la atención del servicio, busque la adición de tratamiento de acuerdo con el promedio de los parámetros de QOL presentadas por los pacientes, siendo estos últimos los usuarios finales de los servicios de salud. Descriptores: calidad de vida; pacientes; epidemiologia.

${ }^{1}$ Acadêmica de Enfermagem da Faculdade de Ciências Médicas da Unicamp. Campinas (SP), Brasil. E-mail: anac4848@yahoo.com.br; ${ }^{2}$ Enfermeira, professora Doutora do Departamento de Enfermagem da Faculdade de Ciências Médicas da Unicamp. Campinas (SP), Brasil. E-mail: katia@fcm.unicamp.br

Artigo elaborado a partir do Trabalho de Conclusão de Curso $<$ Qualidade de vida de pacientes da Unidade de Emergência Referenciada do Hospital de Clínicas da Unicamp >> apresentado ao Curso de Graduação em Enfermagem, do Departamento de Enfermagem da Faculdade de Ciências Médicas da Universidade Estadual de Campinas. Campinas-SP, Brasil. 2010 


\section{INTRODUCÃO}

Na última década a expressão qualidade de vida (QV - Quality of life - QOL) tem se tornado muito frequente na medicina e na área da saúde em geral, sendo que os estudos que abordam essa temática são cada vez mais numerosos. $^{1}$

Atualmente, os fatores externos que interferem na QV do indivíduo tem se tornado foco de estudo do tema. Entre eles, há os fatores estressantes, condição imposta ao indivíduo pela sociedade de consumo, de caráter cumulativo e explosivo, desgastante, que exige sempre alternativas compensadoras. São justamente esses reparos a sobrevivência, exigência natural do corpo e do íntimo do indivíduo, que fazem parte da preocupação natural, entre outras, daqueles que se envolvem com o estudo da QV. Os indivíduos buscam, primeiramente, formas, as mais variadas, para atenuar esse desgaste do dia-a-dia e, até, ter a pretensão de fazer desaparecer os fatores que desencadeiam o estresse. Em contrapartida, essa fragilidade do sujeito entra para a análise dos criadores de produtos e dos especialistas em marketing que the propõem atenuar ou suprir seu cansaço físico ou mental com adoção de fórmulas compensadoras que podem ir desde a aquisição de um imóvel, de um carro, de um alimento de qualidade, até outras ilusões e sonhos. $^{2}$

A dedicação do ser humano ao seu próximo doente, até o início da Era Cristã, era denotada como sinal de bondade e de solidariedade no momento de sofrimento. Durante a Idade Média, período em que a religião católica predominou em todos os sentidos e aspectos da vida do homem europeu, cabia aos homens religiosos a manutenção dos hospitais, e as mulheres, cuidados diretos com o paciente. ${ }^{3}$

A dicotomia entre trabalho manual e chefia (administração) leva, na grande maioria das vezes, o profissional de enfermagem graduado a distanciar-se dos pacientes e, consequentemente, dos problemas do dia-adia dos técnicos e auxiliares de enfermagem que assumem maior contato diretamente com o paciente. Tal realidade pode ser atribuída à escola de Florence Nightingale que repassava às "ladies" (mulheres da alta burguesia britânica), o trabalho de chefia e às "nurses" (enfermeiras advindas de classes menos favorecidas), o trabalho direto com 0 paciente. $^{3}$

Considerando os progressos que a profissão vem alcançando, especialmente após configurar-se como área do saber, tendo se firmado como escola no âmbito da universidade, não podemos restringir a enfermagem apenas aos cuidados com o paciente e a administração. ${ }^{3} 0$ profissional enfermeiro tem responsabilidade profissional, social, moral e científica, amplamente reconhecida, de avaliar e garantir a qualidade dos cuidados prestados aos usuários. ${ }^{4}$

A satisfação de um paciente com um serviço de urgência e emergência abrange diversos aspectos da atenção oferecida. A avaliação de satisfação do paciente pode ser realizada através de aspectos relativos à estrutura do serviço, como atenção, pontualidade, tempo de espera, deslocamento até o hospital, organização do atendimento, cuidado e competência de toda a equipe envolvida (médicos, enfermeiros, auxiliares etc) além de aspectos relacionados especificamente ao atendimento de enfermagem e médico.

O Hospital de Clínicas (HC) da Universidade Estadual de Campinas (UNICAMP) foi escolhido como campo do estudo por possuir um complexo de saúde de referência e excelência, nacional e internacional, que presta assistência médico-hospitalar universal e complexa, desenvolvendo ações de promoção do ensino, da pesquisa e da assistência, constituindo-se em palco de grandes e importantes descobertas. ${ }^{5}$ Dentre as suas especialidades, conta com importantes serviços como a Unidade de Emergência Referenciada (UER). ${ }^{6}$

Assim, nota-se a importância em avaliar o perfil da QV dos pacientes que chegam ao serviço da UER do HC, permitindo que o profissional enfermeiro tenha subsídios para propor melhorias no serviço, especialmente focando na recepção e estadia do paciente, além de outros cuidados envolvendo serviços de psicologia, assistência social entre outros. ${ }^{6}$

Optou-se pelo instrumento para avaliação de QV “The World Health Organization Quality of Life - WHOQOL-bref" devido à sua rápida e fácil aplicabilidade, confiabilidade, validade e responsividade. ${ }^{7-10}$ Não existem, além do mais, registros de estudos que tenham empregado 0 WHOQOL-bref em pacientes atendidos em uma Unidade de Emergência Referenciada.

\section{OBJETIVOS}

- Conhecer a qualidade de vida dos pacientes atendidos da Unidade de Emergência Referenciada (UER) de um hospital universitário (Hospital de Clínicas da 
Universidade Estadual de Campinas) visando futuras ações de melhorias para atendimento deste grupo populacional.

- Caracterizar e analisar a qualidade de vida da população de pacientes atendidos na UER do HC/Unicamp através do instrumento genérico (WHOQOL-bref) e as especificidades da população estudada segundo os domínios: físico, psicológico, meio ambiente e relações sociais.

\section{MÉTODO}

Trata-se de um estudo do tipo transversal, descritivo e analítico baseado em estratégias de análise quantitativa, realizado na sala de recepção de pacientes classificados segundo a classificação de risco da Unidade de Emergência Referenciada do Hospital de Clínicas da Universidade Estadual de Campinas. O projeto foi aprovado pelo Comitê de Ética em Pesquisa da Faculdade de Ciências Médicas da Universidade Estadual de Campinas, parecer $n^{\circ} 1036 / 2009$.

A amostra constitui-se por 100 pacientes que estavam aguardando atendimento na recepção após passarem pela classificação de risco da UER, no período de junho a julho de 2010. Os sujeitos foram recrutados de acordo com a ordem de cores da classificação de risco, obedecendo à ordem de chegada.

Os critérios de inclusão compreenderam idade igual ou superior a 18 anos; aguardar atendimento após ter passado por classificação de risco; ter sido classificado de acordo com as cores: amarelo, verde e azul; capacidade para ler as instruções e responder o instrumento; participação voluntária na pesquisa assinando o Termo de Consentimento Livre e Esclarecido. Foram excluídos sujeitos classificados com a cor vermelha, pois esta requer atendimento imediato, ${ }^{11}$ sem tempo de espera na recepção; sujeitos que não aceitarem participarem voluntariamente da pesquisa; quaisquer sujeitos que não se enquadrarem nos critérios de inclusão.

Assim, seguiu-se o critério de selecionar o sujeito optando prioritariamente pela cor amarela. Na ausência da mesma, optou-se pela verde ou azul, sequencialmente. Após a seleção, os sujeitos foram informados sobre a pesquisa e seus aspectos éticos pelo pesquisador, mediante a apresentação do Termo de Consentimento Livre e Esclarecido, segundo a Resolução 196/96.

Em seguida, aos sujeitos participantes foi aplicado o questionário "The World Health
Organization Quality of Life - WHOQOL-bref, com esclarecimento de quaisquer dúvidas no ato do preenchimento. Este é um instrumento genérico de QV composto de 26 itens pertinentes à avaliação subjetiva do indivíduo em relação aos aspectos que interferem em sua vida nos últimos 15 dias. Este instrumento de medida da QV abrange quatro domíniosfísico, psicológico, relações sociais e meio ambiente.

As variáveis estudadas foram gênero (masculino ou feminino), idade e escala WHOQOL-bref.

Os dados foram analisados descritivamente por frequências absolutas ( $\mathrm{n}$ ) e relativas (\%) para as variáveis categóricas e para as variáveis contínuas usou-se a média, desviopadrão, mediana, primeiro e terceiro quartil, valores máximos e mínimos. A aderência dos dados a distribuição normal foi avaliada segundo o teste de Kolmogorov-Sminrnov. Para a comparação por sexo, o teste t de Student foi utilizado para comparar a idade e o teste de Mann-Whitney para comparar os escores do WHOQOL-bref.

$\mathrm{Na}$ análise dos dados foi utilizado o software SAS versão 9.1.3 (SAS Institute Inc., Cary, NC, USA, 2002-2003) e foi considerado o nível de $5 \%$ de significância estatística.

\section{RESULTADOS}

O estudo transversal da QV dos pacientes atendidos na UER/HC da Unicamp envolveu a coleta de dados epidemiológicos, bem como a aplicação de escala de aferição da qualidade de vida (WHOQOL-BREF). Dos 100 indivíduos que aceitaram participar do projeto, todos preencheram adequadamente o questionário de QV e identificaram o sexo e idade no Termo de Consentimento Livre e Esclarecido.

A população estudada apresentou idade média de 40,0 anos, tendo a faixa etária variado de 18,0 anos a 83,0 anos, fato observado na Tabela 2. Na Tabela 1 os dados demonstram predomínio do sexo feminino na população estudada, $71,0 \%$ mulheres e $29,0 \%$ homens. Também se observa na Tabela 3 dentre os sujeitos participantes uma idade relativamente menor para o sexo feminino, com média de 39 anos, em contrapartida para o sexo masculino, com média de 42 anos. 
Tabela 1. Caracterização da amostra segundo o sexo. Campinas/SP, 2010.

\begin{tabular}{lcc}
\hline Sexo & $\mathrm{n}$ & $\%$ \\
Homens & 29 & 29,0 \\
Mulheres & 71 & 71,0 \\
\hline
\end{tabular}

Tabela 2. Caracterização da amostra segundo a idade. Campinas/SP, 2010.

\begin{tabular}{cccccccc}
\hline & Média & Desvio padrão & Mediana & $1^{\circ}$ Quartil & $3^{\circ}$ Quartil & Mínimo & Máximo \\
Idade & 40,0 & 14,0 & 41,0 & 28,0 & 49,0 & 18,0 & 83,0 \\
\hline
\end{tabular}

Tabela 3. Comparação da idade segundo sexo. Campinas/SP, 2010.

\begin{tabular}{cccccc}
\hline & \multicolumn{3}{c}{ Mulheres } & \multicolumn{3}{c}{ Homens } & Valor $p^{\S}$ \\
Idade & Média & Desvio padrão & Média & Desvio padrão & 0,4039 \\
\hline
\end{tabular}

${ }^{\S}$ Teste de Student.

A Tabela 4 ilustra a média específica obtida em cada domínio (físico, psicológico, relações sociais e meio ambiente) analisado pela escala de aferição da QV. Todos os participantes preencheram devidamente as questões pertinentes aos domínios. As médias encontradas pelos diferentes domínios foram semelhantes, demonstrando certa homogeneidade entre esses aspectos na vida dos sujeitos analisados.

O maior escore dentre os domínios foi alcançado pelo escore psicológico, com média de 66,0 (DP: 17,0). Logo após, os domínios físico e relações sociais com médias respectivas de 63,0 (DP: 18,0) e 63,0 (DP: 24,0). O domínio ambiental obteve escore mais baixo, com médias de 55,0 (DP: 15,0).
O domínio relações sociais foi o que apresentou maior pontuação máxima, alcançando o escore 100. Já o domínio físico obteve a menor pontuação máxima $(96,0)$, e o construto psicológico obteve a menor pontuação mínima $(92,0)$. O domínio ambiental apresentou a menor pontuação $(91,0)$ comparativamente com os outros domínios pesquisados. Esse domínio tem relação com algumas necessidades humanas básicas quando relaciona segurança física e proteção, ambiente no lar, recursos financeiros, cuidados de saúde e sociais (disponibilidade e qualidade), oportunidade de adquirir novas informações e habilidades, participação em oportunidades de recreação e de lazer, ambiente físico (poluição/ruído/trânsito/clima) e transporte.

Tabela 4. Análise descritiva dos domínios do Whoqol-Bref. Campinas/SP, 2010.

\begin{tabular}{lrcccccc}
\hline Domínios & Média & Desvio padrão & Mediana & $1^{\circ}$ Quartil & $3^{\circ}$ Quartil & Mínimo & Máximo \\
Físico & 63,0 & 18,0 & 68,0 & 54,0 & 75,0 & 18,0 & 96,0 \\
Psicológico & 66,0 & 17,0 & 69,0 & 54,0 & 79,0 & 4.2 & 92,0 \\
Relações & 63,0 & 24,0 & 67,0 & 50,0 & 83,0 & 0,0 & 100,0 \\
sociais & 55,0 & 15,0 & 56,0 & 45,0 & 66,0 & 13,0 & 91,0 \\
Ambiental & 5,0 & & & &
\end{tabular}

Com relação à avaliação da QV entre os gêneros, o sexo feminino apresentou melhor QV nos domínios psicológico, relações sociais e ambiental do que o masculino. 0 sexo masculino obteve melhor $\mathrm{QV}$ do que o sexo feminino somente no domínio físico. Comparando-se o domínio físico nos gêneros $(p<0,3749)$, observamos que $o$ gênero feminino atingiu média de 64 enquanto que o masculino obteve média de 68. Ao analisar o domínio psicológico em relação ao sexo feminino e masculino $(p<0,0203)$, temos uma média para o sexo feminino de 75 e uma média de 67 ao sexo masculino. Já a relação do domínio social com os gêneros $(p<0,2225)$ demonstrou pontuação média de 67 no gênero feminino, e de 58 no masculino. Da mesma forma, foi comparado o domínio ambiental com os gêneros $(p<0,0544)$, revelando média de 59 para o sexo feminino, bem como uma média de 53 para o masculino.

\section{DISCUSSÃO}

Com relação à análise dos diferentes domínios, foi constatado que o domínio psicológico apresentou pontuação máxima, e o domínio ambiental obteve escore mínimo. Contudo, as médias não apresentaram diferenças significativas, tendo obtido escores semelhantes entre si.

Observamos a partir dos dados obtidos na escala que avaliou os quatro domínios uma baixa QV em todas as dimensões, pois os escores estão abaixo da média obtida. Estudo transversal em pacientes institucionalizados em um hospital psiquiátrico evidenciou baixos escores em todos os domínios: físico $(14,65)$, 
psicológico (16), relações social $(20,2)$ e meio ambiente $(17,1) .^{12}$

Reafirma-se então, pelos dados já descritos anteriormente, que os agravos ambientais estão possivelmente interferindo na QV dos pacientes atendidos na UER/HC, pois 0 domínio ambiental apresentou o menor escore $(55,0)$. Os aspectos ambientais incluem necessidade de segurança física e proteção, ambiente no lar, recursos financeiros, cuidados de saúde e sociais, oportunidade de adquirir novas informações e habilidades, participação em oportunidades de recreação e de lazer, ambiente físico e transporte. Em grupos de pacientes com lesões medulares, encontrou nos domínios meio ambiente e físico os piores escores de avaliação, respectivamente 49,51 e 50,26, pelo fato da lesão medular causar alterações físicas limitante nas funções motoras. ${ }^{13}$

Outro estudo avaliou a gravidade da dependência do álcool na QV, em pacientes dependente de álcool do sexo masculino, e o escore para o domínio da relação sociais e meio ambiente foram os piores encontrados, $(10,8$ e 10,56) observando que nos pacientes com maior dependência de álcool há pior QV. ${ }^{14}$

Os resultados encontrados são preocupantes, pois dentro do domínio ambiental encontra-se o acesso aos serviços de saúde, envolvendo a disponibilidade e qualidade desses. No fluxo de atendimento nos serviços de saúde, os pacientes saudáveis ou com doenças crônicas devem realizar acompanhamento regular nas unidades básicas de saúde (UBS) ou ambulatórios e, através desses, serem encaminhados a serviços especializados e de maior complexidade de acordo com as demandas que apresentem. Em casos de emergências e urgências os pacientes devem procurar serviços de atendimento imediato, os pronto-socorros (PS), como no caso do local da pesquisa. ${ }^{15}$

Entretanto, a teoria não é observada no local do estudo, realidade semelhante à observada em pesquisa sobre a interação entre profissionais e pacientes em um PS. 0 déficit de conhecimento da população acerca desse fluxo, multiplicado pelas condições prejudicadas apresentadas pelos serviços primários, levam os pacientes a procurarem os serviços de PS para satisfazer suas necessidades. ${ }^{15}$

Por outro lado, os serviços de PS também sofrem com a falta de recursos humanos e materiais, com estruturas físicas inadequadas para a realidade enfrentada, por atenderem com "portas abertas", ou seja, todos os que buscam assistência são atendidos, independente das condições em que se encontrem os serviços. ${ }^{16}$

Com a problematização exposta, o número excessivo de pacientes (demanda inadequada para as características do atendimento), condições físicas, escassez de recursos humanos e materiais, e o acesso irrestrito, geram grande sobrecarga dos funcionários que atendem nestes serviços, diminuindo consideravelmente a qualidade da assistência prestada, ou seja, o profissional torna-se limitado cumprindo seu papel instrumental, esquecendo do atendimento humanizado, ao assimilar-se à rotina da unidade. ${ }^{15}$

Verifica-se, portanto, que a desorganização do sistema de saúde, em toda sua hierarquia, acaba por refletir em queixas dos pacientes atendidos nos serviços de PS no país, evidenciada pelo baixo escore do domínio ambiental no instrumento sobre QV do presente estudo.

Em relação ao domínio psicológico, este obteve maior escore $(66,0)$. Tal domínio engloba aspectos de frequência de sentimentos positivos/negativos consigo mesmo, o pensar, o aprender, a memória e a concentração, a auto-estima, imagem corporal e aparência e espiritualidade/religião/crenças pessoais. Embora o desenho deste estudo não esclareça detalhes sobre o suporte psicológico, supõe-se que os pacientes estudados apresentem o suporte psicológico necessário, já que obtiveram escores satisfatórios no domínio psicológico. Estudo envolvendo familiares de pacientes de uma Unidade de Terapia Intensiva obteve escores altos nos domínios físico e psicológico, e escore baixo nos domínios relações sociais e meio ambiente. ${ }^{1}$

Os domínios físico e relações sociais obtiveram escores iguais $(63,0)$. O domínio relações sociais corresponde ao apoio de amigos, vida sexual e relacionamento com as pessoas próximas ou não. Já o domínio físico abrange questões ligadas à dor e conforto, energia e fadiga, sono e repouso, mobilidade, atividades da vida cotidiana, dependência de medicações ou de tratamentos e capacidade para o trabalho.

Observa-se que apesar do domínio físico conter questões relacionadas ao acesso aos serviços de saúde (medicações e tratamentos), este não apresentou baixo escore, pelo contrário, obteve a menor pontuação máxima $(96,0)$.

Isto se explica talvez pelo fato do problema de atendimento de saúde não está nas 
técnicas realizadas pelos profissionais e sim pelo atendimento desumanizado. A presença da mecanização do cuidado, uma dicotomia entre a realização de procedimentos e o estar com o paciente. Para os profissionais do PS, o cuidar, devido ao local de trabalho, poderia ser resumido aos procedimentos realizados. ${ }^{15}$

Assim, podemos inferir sob a óptica dos pacientes que o que interfere no atendimento de saúde pelos profissionais, no âmbito do atendimento de urgência e emergência num $\mathrm{PS}$, é a rotina viciosa, que torna obscura a percepção dos profissionais, traduzida pela maior valorização do fisiológico e uma exclusão do ser psicossocial e psicoespiritual. Isto não deve ocorrer, já que tais características não são autônomas e, como profissionais, é necessário considerá-las durante todo o serviço, ou seja, como o paciente se comporta, o que ele sente e pensa. $^{17}$

Já em relação à comparação dos domínios entre gêneros, estudos realizados comparando os gêneros comprovaram que a QV no domínio físico é melhor em homens do que em mulheres, ${ }^{18-19}$ fato observado na pesquisa. Isso pode estar relacionado a diferenças nas condições de vida ou a diferenças na percepção da QV entre homens e mulheres, ou seja, os dois sexos podem ter condições de vida semelhantes, porém, formas diferentes para analisar e ponderar diversos aspectos de sua vida. Porém, a QV maior nos domínios psicológico, ambiental e relações sociais entre as mulheres do que em homens está em contraposição aos resultados desses estudos.

Há de se observar nos resultados as diferentes faixas etárias que responderam ao instrumento, porém o estudo não objetiva comparar a relação existente entre domínios e faixas etárias, embora existam estudos que descrevem a relação direta de QV por faixas etárias. $^{20}$

Os dados descritos também sugerem que a utilização do instrumento de medida da QV pode ser uma maneira eficaz de acompanhamento e detecção de alterações na QV privilegiando a opinião dos pacientes e permitindo uma abordagem multidisciplinar, almejando ações de promoção, proteção e recuperação da saúde.

\section{CONCLUSÃO}

Consideramos que médias baixas de satisfação da QV em todos os domínios denotam preocupação com os pacientes atendidos na UER/HC, principalmente por representarem os usuários que procuram atendimento em um PS. A literatura demonstra o grande fluxo de pacientes, que deveriam ser atendidos em UBS sendo direcionados aos serviços de atendimento de urgência e emergência, com condições físicas inadequadas e déficit de recursos humanos para atendimento, seja por problemas estruturais, seja por desconhecimento da população acerca da função do serviço. ${ }^{15-17}$

Nos serviços de urgência e emergência, o paciente com baixa QV depara-se com profissionais - médicos e enfermeiros, cujo atendimento é centralizado na resolução rápida da queixa principal e nos procedimentos a serem realizados, resumindose a estes todo o cuidado a ser prestado ao paciente. Há uma deformação no conceito de comunicação, sendo identificada como o domínio verbal utilizado para comunicar ao paciente os procedimentos que the serão realizados ou para desmistificar dúvidas. Assim, nas instituições hospitalares, a qualidade de vida deve focar também nas condições para a qualidade do trabalho, nas estratégias para alterar, melhorar ou implementar ações que garantam condições adequadas e atendimento profissional competente e responsável. ${ }^{21}$

Esta pesquisa identificou que a qualidade do serviço de saúde de urgência e emergência deficitária prestada ao paciente reflete diretamente na sua QV, tornando-a relativamente baixa, à medida que um fator interfere diretamente nos outros, através dos domínios ambiental, físico, meio ambiente e psicológico. Apesar das limitações identificadas, a aplicação do instrumento de QV WHOQOL-bref mostrou-se fidedigna para a população estudada. $\mathrm{Na}$ pesquisa, tal instrumento visa, sobretudo, a evolução da promoção da saúde e da QV dos indivíduos.

Os problemas identificados são de grande complexidade. Não há julgamento compatível a nenhum dos lados - do paciente e do serviço, já que ambos estão prejudicados. Somente alterações no âmbito nacional visando à conscientização da população e a reorganização dos recursos, poderiam resolvêlos.

\section{REFERÊNCIAS}

1. Zanei SSV. Análise dos instrumentos de avaliação de qualidade de vida WHOQOL-bref e SF-36: confiabilidade, validade e concordância entre pacientes de Unidades de Terapia Intensiva e seus familiares [tese de doutorado]. São Paulo (SP): Escola de Enfermagem da Universidade de São Paulo; 
2006. 134 p.

2. Constantino MAC. Avaliação da qualidade de vida: desenvolvimento e validação de um instrumento, por meio de indicadores biopsicossociais, junto à Comunidade da Universidade de São Paulo - USP [tese de doutorado]. São Paulo (SP). Programa de Nutrição Humana Aplicada (FCF/FEA/FSP): 2008. 163 p.

3. Aquino KS. Proposta para a qualidade em saúde, visando ao paciente com traumatismo cranioencefálico, em vista de acidentes de trabalho e o papel do enfermeiro em sua recuperação do HC - Unicamp [tese de doutorado]. Campinas (SP): Departamento de Ciências Médicas da Faculdade de Ciências Médicas da universidade Estadual de Campinas; 1999. 149 p.

4. Dreyer E. Definição operacional da qualidade da assistência de enfermagem em Unidade de Terapia Intensiva de um Hospital Escola. II Encontro de Administração em Enfermagem, 1997.

5. Hospital de Clínicas. Faculdade de Ciências Médicas. Universidade Estadual de Campinas. [Citado 2009 set 24]. Disponível em: www.hc.unicamp.br/especialidades

6. Duram ECM. Capacidade para o trabalho entre os trabalhadores de Enfermagem do Pronto-Socorro de um Hospital Universitário [dissertação de mestrado]. Campinas (SP): Faculdade de Ciências Médicas da Universidade Estadual de Campinas: 2002, 100 p.

7. Fleck MPA, Fachel O, Louzada S, Xavier M, Chachamovich E, Vieira G, et al. Desenvolvimento da versão em português do instrumento de avaliação de qualidade de vida da Organização Mundial da Saúde (WHOQOL100). Rev Bras Psiquiatr 1999a; 21(1):19-28.

8. Fleck MPA, Leal OF, Louzada S, Xavier M, Chachamovich E, Vieira $G$, et al. Desenvolvimento e aplicação da versão em português do instrumento de avaliação de qualidade de vida da organização mundial da saúde (whoqol-100). Revista HCPA 1999b; 19(1):9-30.

9. The WHOQOL Group. Development of the World Health Organization WHOQOL-bref Quality of Life Assessment 1998. Psychol Med 1998; 28:551-8.

10. Fleck MPA, Leal OF, Louzada S, Xavier M, Chachamovich E, Vieira G, et al. Aplicação da versão em português do instrumento abreviado de avaliação da qualidade de vida “WHOQOL- bref”. Rev de Saúde Pública. 2000; $34(2): 178-83$,.
11. Manual dos Processos de Trabalho (Normas e Rotinas) da Unidade de Emergência Referenciada. Versão 2008. 31p.

12. Wagner CL, Fleck APM. Avaliação da Qualidade de na Esquizofrenia. In: Fleck APM e Colaboradores. A Avaliação de Qualidade de Vida: Guia para Profissionais da Saúde. 1. ed. Porto Alegre: Artmed; 2008. p. 28

13. Bampi SNL, Guilherm D, Lima DD. Qualidade de vida em pessoas com lesão medular traumática um estudo com 0 WHOQOL- bref. Rev Bras Epidemiologia. 2008; 11(1):67-77.

14. Lima SBFA, Fleck APM. Qualidade de vida e alcoolismo. In: Fleck APM e Colaboradores. A Avaliação de Qualidade de Vida: Guia para Profissionais da Saúde. 1. ed. Porto Alegre: Artmed, p. 28, 2008

15. Souza RB, Silva MJP, Nori A. ProntoSocorro: uma visão sobre a interação entre profissionais de enfermagem e pacientes. Revista Gaúcha de Enfermagem 2007; 28(2):242-9.

16. Galloti RMD. Eventos adversos e óbitos hospitalares em serviço de emergência clínicas de um hospital universitário terciário: um olhar para a qualidade da atenção [dissertação de Mestrado]. São Paulo: Faculdade de Medicina, Universidade de São Paulo; 2003. $148 \mathrm{f}$.

17. Silva AL, Nascimento KC, Virgílio MS, Mendonça RS. Análise dos fatores de cuidado de Watson em uma unidade de emergência. Revista Gaúcha Enfermagem 2002;23(2):16-26.

18. Lipp MEN, Tanganelli MS. Stress e qualidade de vida em Magistrados da Justiça do Trabalho: diferenças entre homens e mulheres. Psicol Reflex Crit 2002; 15(3): 537 548.

19. Pinheiro AG, Bianchini TMQ, Campos W, Petroski EL. Domínio Físico da Qualidade de Vida entre Adolescentes: Associação com Atividade Física e Sexo . Rev. salud pública [online]. 2009; 11(1):50-61.

20. Chepp CC. Estudo transversal da qualidade de vida através da escala WHOQOL-Bref da população octogenária e nonagenária de Siderópolis. Monografia (Graduação em Medicina). Criciúma (SC): Curso de Medicina na Universidade do Extremo Sul Catarinense/UNESC. 2006. 52 p.

21. Souza MA, Stancato K. Quality of life assessment of healthcare professionals of the Clinics Hospital of Unicamp. Rev enferm UFPE on line [periódico na internet]. 2011 jun [acesso em 2011 out 10];5(4):886-95. 
Disponível

em:

http://www.ufpe.br/revistaenfermagem/inde

x.php/revista/issue/view/42

Sources of funding: No

Conflict of interest: No

Date of first submission: 2011/10/10

Last received: 2012/01/21

Accepted: 2012/01/22

Publishing: 2012/02/01

\section{Corresponding Address}

Ana Carolina Gaban

Cidade Universitária Zeferino Vaz

Rua Tessália Vieira de Camargo, 126

Caixa Postal 6111

CEP: 13083-887 - Campinas (SP), Brazil 